\title{
Summation of successively established orientation-contingent color aftereffects
}

\author{
KEITH D. WHITE \\ Hunter Laboratory of Psychology, Brown University, Providence, Rhode Island 02912
}

\begin{abstract}
Orientation-contingent color aftereffects (CAEs) were studied using a color-cancellation technique for measurement. Procedures involved more than one period of inspection, each of which established CAEs, carried out successively to produce a combined or net CAE (akin to "nullification" used in other studies). When opposite color-orientation pairings were used in successive inspections, the net CAE was predicted faithfully by summation of the constituent CAEs as measured from each period of inspection independently, and thus showed qualitative as well as quantitative changes in coloration over time. This implies that "nullification" does not truly eliminate CAEs as has previously been assumed, and suggests that the units of measure used here may be linearly representative of CAE strengths. When the successive inspections used identical color-orientation pairings, however, summation was poor. This can be explained if inspection alters the mechanisms underlying CAEs, rendering retention of a successively established CAE of the same kind less effective.
\end{abstract}

Orientation-contingent color aftereffects (CAEs) are relatively long-lasting perceptual changes that are revealed as subjective coloration on particular black-and-white test patterns. The present studies use a color-cancellation technique to standardize and systematize judgments of CAE vividness, providing measures in physical units of chromaticity from which the subjective colorations may be deduced. The main problem addressed by these studies is that of devising quantitatively useful estimates of the CAE strengths from the units of chromaticity. To that end, three experiments have tested the hypothesis that the subjects' CAEs and the manner of their assessment behave as a linear system.

The procedures make use of more than one period of inspection, each of which establishes CAEs, but they are carried out successively to produce a combined or net CAE. The experimental question is whether the net CAE can be faithfully predicted by summation of the constituent CAEs, as measured from each period of inspection independently. Predictive validity of the summation rule gives evidence on the linear system hypothesis. Successive procedures are also akin to "nullification" and "reversal" paradigms as used in earlier investigations.

Supported by a NSF predoctoral fellowship and by USPHS Grant EY 00774 to Dr. L. A. Riggs. I thank Drs. L. A. Riggs, B. R. Wooten, P. D. Eimas, M. Glickstein, C. S. Harris, D. Skowbo, and C. F. Stromeyer III for improving the manuscript. This paper is based on a thesis submitted in partial fulfillment of the requirements for the Doctor of Philosophy degree at Brown University. The author's present address: Department of Psychology and Center for Sensory Studies, University of Florida, Gainesville, Florida 32610.
Results demonstrate the usefulness of a linear summation rule for predicting certain of the net CAEs. In these cases, the combined CAEs showed both qualitative and quantitative changes in coloration over the time elapsed after inspections, as expected. These findings imply that (1) "nullification" does not truly eliminate CAEs as has been assumed previously, and (2) the units of chromaticity used here may be linearly representative of CAE strengths. In other situations, namely when the successive inspections used identical color-orientation pairings, simple summation yielded poor predictions. These summation failures can be explained if initial inspection alters the underlying mechanisms of CAEs, rendering retention of any successively established identical CAEs less effective.

The perceptual aftereffects described by McCollough (1965) are relatively long-lasting changes in the appearance of certain patterns; they are colored aftereffects that, once established, can be seen on some stimulus patterns but are not readily seen on others. Notable features of these perceptual changes distinguish them from such classic visual aftereffects as negative afterimages. One distinction is reflected in their descriptive name, orientationcontingent color aftereffects (CAEs). The appearance of CAEs depends to an unusual degree on pattern attributes, such as the orientations of lines used to establish or to test them. General methods that are still widely used for demonstrating these pattern-contingent aftereffects were originated by McCollough. The CAEs are established by a few minutes of inspection of two colored patterns presented alternately; for example, one pattern is a grating composed of red and black lines in a single 
orientation and the other pattern, a grating of green and black lines in the orthogonal orientation. Following establishment by inspection, the presence of CAEs may be revealed when the subject views black and white test lines. These appear to be faintly colored rather than neutral, the hue seen as aftereffect on a given test orientation being nearly complementary to the hue with which that orientation had been paired during the inspection period (McCollough, 1965). Yet, perhaps the most striking distinction of CAEs is their time course for decay; they can often be revealed in tests made many hours or even days after their establishment.

These and other findings on CAEs have led to considerable experimental interest, but also to a diversity of theoretical accounts. Models range in their emphasis from those which involve physiological mechanisms of pattern vision, on the one hand (e.g., Creutzfeldt \& Heggelund, 1975; Harris \& Gibson, 1968), to those which postulate factors of learning and memory, on the other (e.g., Mayhew \& Anstis, 1972; Murch, 1976). Yet another class of models attempts to combine relative contributions from perceptual and conditioning processes (Montalvo, Note 1). In the conclusion of their recent review paper, Skowbo, Timney, Gentry, and Morant (1975) suggest that those relative contributions may come to be understood through quantitative operational descriptions of CAEs.

Earlier works most relevant to that aim have used colorimetric testing procedures to secure more nearly objective assessments of CAE strengths. (See for literature review, Anstis, 1975; Skowbo et al., 1975; Harris, Note 2; Stromeyer, Note 3.) Studies of that kind are similar in inspection procedure to McCollough's (1965), but differ with regard to testing. Exemplars of the colorimetric test procedures are color-matching and color-cancellation, other forms of test having aspects of each procedure (e.g., MacKay \& MacKay, 1975). In color-matching, the subject views an achromatic grating on which one of his CAEs is revealed, then adjusts the chromaticity of an adjacent homogeneous field so that it appears the same color as the seen aftereffects (Skowbo, Gentry, Timney, \& Morant, 1974). In colorcancellation, the test grating itself is adjusted in chromaticity until it appears subjectively neutral in color (Fidell, 1970; Sigel \& Nachmias, 1975) or until it matches a reference field (Riggs, White, \& Eimas, 1974). Cancellation can potentially eliminate the subject's awareness of changes in the CAE vividness by requiring that the test pattern always look the same, in the presence as well as in the absence of seen aftereffects. It is in this regard, namely the goal of achieving identical test pattern appearances whether the CAEs are vivid or weak, that color-cancellation judgments are more nearly "Class A" observations (Brindley, 1970).
Systematizing the manner of testing in these ways can allow valid comparisons of results from different individuals and even from different laboratories by the use of standardized chromaticity units to score the color judgments. Colorimetric tests are therefore a powerful empirical tool. Nevertheless, colorimetric tests give only indirect measures, since the CAEs per se are a state of the subject's nervous system. Evidence that CAEs are present comes from altered color reports but, of course, the reports are not the aftereffects themselves. Theoretical inferences about CAE strengths based on chromaticity scores of the subject's color reports are constrained in two important ways. First, color judgments of chromatic lights depend on many factors in addition to the presence of CAEs, the absence of appropriate controls for them serving to alter the nature of the measuring instrument itself (i.e., the subject's particular abilities for reporting color). (See, for secondary sources, Committee on Colorimetry, O.S.A., 1953; Evans, 1948; Graham, 1965; Wyszecki \& Stiles, 1967.) Secondly, chromaticity scores must be expressed in a way that yields quantitatively useful estimates of the strengths of the CAEs themselves. Although there are many standard indices of chromaticity by which to assign numerical scores to the test results, it is not clear how those numbers represent the actual amounts of the aftereffects. There would be little debate that as the subjective colorations become more vivid, such measures as the colorimetric purity (or the subjective correlate, saturation) increase in an ordinal fashion. But does each unit of purity represent the same amount of CAE?

The present experiments have two main purposes which can be distinguished as having practical or theoretical impacts. The practical question is whether the "neutralization" procedure, in which subjects are exposed to red and to green gratings of the same orientation in successive periods of inspection, accomplishes the desired goal of eliminating CAEs (see, e.g., Stromeyer, 1969). Because the CAEs are so long-lasting, it would be useful to have a way to get rid of them, and colorimetric tests can potentially verify whether this "neutralization" procedure eliminates or otherwise renders the CAEs ineffective.

The question of theoretical impact is more detailed and quantitative, seeking evidence on how particular chromaticity scores represent CAE strengths. It asks whether useful estimates of CAE strengths can be made from the particular colorimetric index used for scoring in our laboratory (e.g., White, 1976), or alternatively whether a different numerical scaling (such as logarithmic transformation) or even a different index might yield more representative strength estimates. Useful estimates of CAE strengths can be defined as the ones that allow straightforward predictions of the subject's responses. A simple but 
feasible rule, algebraic addition, is used here to calculate specific predicted values from previously measured chromaticity scores (the empirical estimates of CAE strength). To test these predictions, and hence to test the hypothesis of linear summation for our empirical CAE strength estimates, the experiments employ a paradigm in which repeated periods of inspection are used to establish a net CAE. The experimental question is whether the combined or net CAE can be predicted by simply adding together CAEs that had been measured in isolation for each constituent period of inspection. The extent of predictive validity gives important evidence on the usefulness of our numerical scores as quantitative estimates of CAE strengths.

The rule of algebraic addition is a straightforward one, but its use requires supplementary assumptions of linearity. Thus, a more specific statement of the hypothesis is that the subject's CAEs behave as a linear system when measured by the particular test procedure used here. A linear system may be defined by the properties of (a) a state-invariant response function, and (b) superposition of causes and effects. The "system" observed has as its components both the subject and the test method. If this system behaves as a linear one, thus confirming the summation rule, it implies that (1) the CAEs established by means of separate periods of inspection must combine as though the inspections were independent (superposition of causes and effects), and (2) the colorimetric scores used as a dependent measure must be approximately linearly related to the net CAE strengths, in order that the measured net response functions be in quantitative agreement with the predictions. Quasi-linear behavior can be mimicked by particular, mutually offsetting nonlinearities in the system components (the subject and the test method), but such an arrangement is not a parsimonious account of observed linearity. On the other hand, if linear system behavior were not to be found, that fact alone does not lead to a crisp conclusion regarding the source of the nonlinearity. The fundamental properties of $a$ linear system ( $a$ and $b$ above) can be reduced for purposes of calculation to a simple rule of algebraic summation, the predictive validity of which is evaluated in the present experiments.

The interested reader can calculate from equations given in the Appendix that linear system predictions must fail for particular limiting conditions of the present experiments, for example, when zero time elapses between successive periods of inspection. The predictions nevertheless describe suitably well the results of Riggs et al. (1974), for which several thousand minutes had elapsed between successive inspections. The present experiments utilize intermediate lengths of time between the inspections. These durations are long relative to the periods of inspection but short relative to the persistence of CAEs; hence, the inspections may act more nearly independently, while the CAEs established by them can each be present with sufficient strength to be measured in combination.

\section{EXPERIMENT 1}

In the first experiment, combined CAEs are established for each subject by means of two successive periods of inspection. The initial inspection period (11.25 min) is followed by an interval of testing and of "natural" stimulation during which the CAEs undergo partial decay (the interinspection interval). Test made during the interinspection interval assess the CAEs that were built up by the initial inspection alone. The duration of the interinspection interval (43 or $91 \mathrm{~min}$ ) is short relative to CAE persistence, so the initial CAEs are still present with considerable strength when the second period of inspection $(5.00 \mathrm{~min})$ is carried out to build up additional CAEs. Tests made following the second inspection show the combined influences of CAEs that had been established during both of the inspection periods; results of those tests give evidence regarding the hypothesis.

Quantitative predictions from the summation rule involve three main steps: (1) Simple descriptive functions are fitted by the method of least squares to the results of Riggs et al. (1974) and White (1976). (2) The particular functions that correspond to conditions used here are added algebraically. (3) The value of one free parameter is estimated, namely a factor of proportionality whose value is characteristic for each subject. Previous studies in our laboratory and others have reported significant individual differences in the absolute chromaticity scores (e.g., MacKay \& MacKay, 1974; Riggs et al., 1974; Skowbo et al., 1974). (See the Appendix for details of calculation.)

\section{Method}

Subjects. Four males (K.W., S.E., D.H., B.C.) and three females (A.P., H.B., J.M.) with normal corrected acuity served as subjects. Three were experienced observers (K.W., S.E., A.P.); two were initially naive, paid volunteers (B.C., H.B.); and the others were inexperienced but informed of the expectations.

Apparatus. The equipment used for establishment and testing of the CAEs has been described in detail by White (1976). Its main purposes are (a) to project alternately, in high-purity complementary colors, line grating inspection patterns used for building up the CAEs, and (b) to provide test gratings that are variable in chromaticity. The test pattern consists of a bipartite field, one half of which is a $45^{\circ}$ grating and the other half a $135^{\circ}$ grating, projected by a special color mixing device. The midpoint of adjustment on this device provides achromatic test gratings in both halves of the field by suitable mixtures of complementary lights. Other adjustments produce differences in chromaticity between test field halves by illuminating the test gratings with various mixtures of the complementary lights. At each point of adjustment, the excitation purity $\left(P_{e}\right)$ of magenta in one halffield is nearly the same as the $P_{e}$ of green in the other half-field. The color mixing device has a scale marked in convenient units that are proportional to $\mathrm{P}_{\mathrm{e}}$. The scale displays positive and negative values, the sign of a score indicating which color is predominant on a particular test grating (see White, 1976, for details).

For the present experiments, the equipment was modified by inserting a Dove prism and artificial pupil into the optical path for the subject's left eye. This arrangement provided optical image rotation which was used to change the orientations of the inspection patterns. ${ }^{1} \mathrm{~A}$ baffle occluded the right eye.

Procedure. Each subject participated individually in two experimental sessions, separated by several days to minimize carryover of the CAEs. Each experimental session consisted of five steps: (1) a pretest, (2) the first period of inspection (11.25 $\mathrm{min}$ ), (3) three test sessions carried out during the interinspection interval (43 or $91 \mathrm{~min})$, (4) the second period of inspection $(5.00 \mathrm{~min})$, and (5) five test sessions carried out after the second inspection.

Every pretest and test session was preceded by at least $1 \mathrm{~min}$ of 
light adaptation to a luminance nearly the same as the test pattern. For each test judgment, assessed CAE strength is scored by the value of $P_{e}$ that the subject adjusts so as to cancel out the colors of his CAEs, rendering the appearance of the test gratings nearly neutral as well as matched in color. This judgment is called a "null match for color." Each test session comprised 10 judgments by the subject of a null match for color on orthogonal $\left(45^{\circ}\right.$ vs. $135^{\circ}$ ) test gratings. A test session required about $3 \mathrm{~min}$ to complete. The experimenter recorded the signed score of $P_{e}$ and the time when each judgment was made. Pretest scores indicate any small color biases on the test pattern prior to building up the CAEs.

The first period of inspection had a duration of $11.25 \mathrm{~min}$, during which two high-purity colored gratings (e.g., $45^{\circ}$-magenta, $135^{\circ}$-green) were presented alternately. The inspection patterns alternated every $5 \mathrm{sec}$, exchanging smoothly with no dark interval between them. Detailed description of these patterns is available elsewhere (White, 1976).

The interinspection intervals lasted about $43 \mathrm{~min}$ for three subjects and about $91 \mathrm{~min}$ for four subjects. The interval could vary by a few minutes from the target value (10\% or less) due to unavoidable circumstances, so the specific expectations were calculated for each experimental sequence in order to account for these unavoidable variations. Exact durations for the interinspection intervals are shown in Results. The particular test sessions which took place during the interinspection interval assessed the CAEs built up by the first inspection.

The second period of inspection had a duration of $5.00 \mathrm{~min}$, likewise being alternate presentations of two high-purity colored gratings, but carried out in one of two possible modes. The mode of the second inspection was determined by the particular pairings of color with orientation on the inspection patterns. Following the example above, SAME mode used the same color-orientation pairings that were used in the first period of inspection (i.e., $45^{\circ}$-magenta, $135^{\circ}$-green), whereas OPPOSITE mode exchanged those color-orientation pairings (i.e., $45^{\circ}$-green, $135^{\circ}$-magenta). Each subject participated in one sequence using SAME mode and another using OPPOSITE mode for the second inspection. Test sessions carried out after the second inspection period give evidence regarding the summation rule.

\section{Results}

Figure 1 compares the observed scores for CAE strength to the curve of expected decay, for subject $K$.W. The graph shows changes in the index of CAE strength, in units of excitation purity $\left(P_{e}\right)$ for the null matches, as a function of time elapsed after the 11.25-min (first) inspection alone. Each plotted point represents the mean $P_{e}$ for 10 judgments of a null match for color, obtained at the mean time shown on the abscissa. The mean $P_{e}$ of pretest judgments was subtracted from each point in that particular experimental session before plotting, in order to discount the subjects' small biases for color found prior to the CAE establishment. Vertical bars mark off one standard deviation of the test judgments on either side of their mean. The points shown represent 520 judgments obtained in 11 different experimental sequences which took place over a period of about 1 year.

Results in Figure 1 indicate the decline over time in K.W.'s scores when no second inspection had occurred, for a period of $200 \mathrm{~min}$ following CAE establishment. It is clear that the general shape of decline can be found reliably. Results for later decay

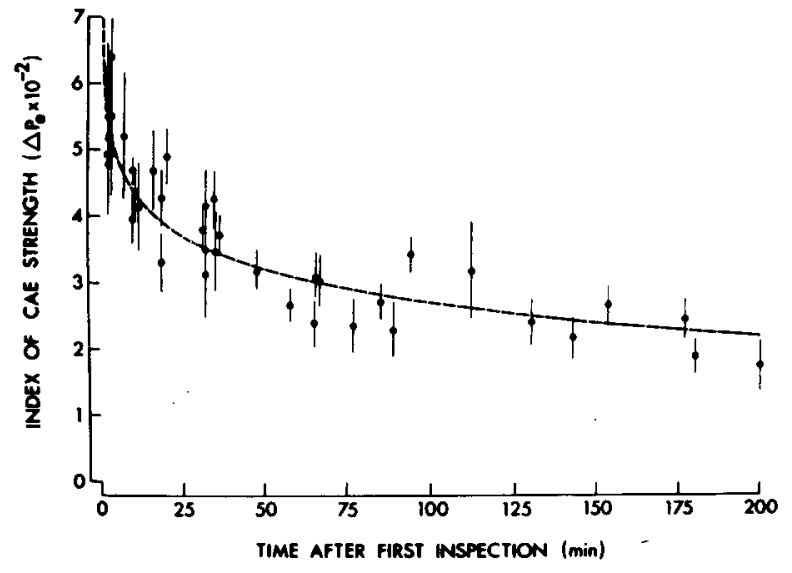

Figure 1. Index of CAE strength shown as a function of time after the first inspection. This graph compares the results from 11 separate sessions of the initial (11.25-min) inspection alone, for subject K.W. Each point plots the mean and SD of excitation purity $\left(P_{e}\right)$ for 10 judgments. The dashed curve represents the expected course of decline as described in text and in the Appendix.

times (not shown) are qualitatively like these. The dashed line drawn through the points represents the expected course of decay as described by Riggs et al. (1974). The data generally lie near the expected decay curve, thus demonstrating it to be an adequate (though not perfect) descriptor.

Figure 2 compares K.W.'s scores to the hypothetical curves for each experimental sequence in which a second period of inspection occurred. Each plotted point represents the same measures as in Figure 1 , the mean $\mathrm{P}_{\mathrm{e}}$ and standard deviation for 10 null match judgments. Different symbol types represent the mode of the second inspection. Closed symbols plot the results from the SAME mode experimental sequence (i.e., in both periods, the subject inspected $45^{\circ}$-magenta, $135^{\circ}$-green), and open symbols plot results from the OPPOSITE mode sequence (i.e., in the second period, the subject inspected $45^{\circ}$-green, $135^{\circ}$-magenta). The times at which the second inspections were carried out are indicated approximately by the positions of the arrows (filled arrow = SAME mode, open arrow = OPPOSITE mode). The interinspection intervals for K.W. lasted $87 \mathrm{~min}$ for the SAME mode sequence and $90 \mathrm{~min}$ for the OPPOSITE mode sequence. Three curves are shown in Figure 2. The dashed line is the same curve shown in Figure 1, representing the expected decay following the first inspection alone. The solid curves are hypothetical, calculated by the algebraic sums of appropriate CAE strength estimates. The upper curve represents Equation $6 \mathrm{a}$ (addition) and the lower curve Equation 6b (subtraction), given in the Appendix.

Consider the SAME mode experimental sequence shown by the closed symbols. Prior to the second 


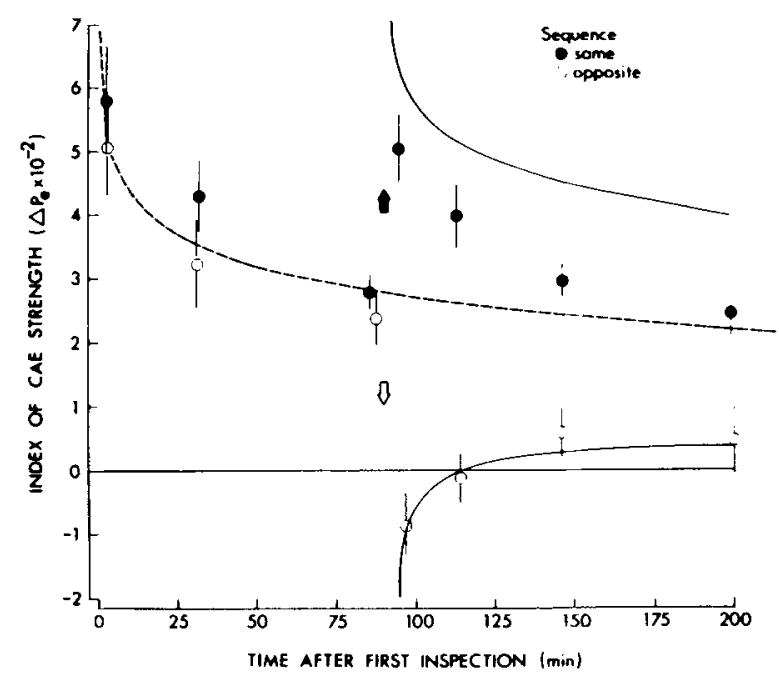

Figure 2. Index of CAE strength shown as a function of time after the first inspection. This graph presents the results from SAME and OPPOSITE mode experimental sequences for subject K.W. Each experimental sequence required two periods of inspection; the mode of inspections is described in text. The times at which the second inspection periods were carried out are indicated by arrows on the figure (filled arrow, SAME mode sequence; open arrow, OPPOSITE mode sequence). Each point plots the same measures as in the previous figure (mean and SD of $P_{e}$ for 10 judgments). Filled symbols present data from the SAME mode sequence, while open symbols present those from the OPPOSITE mode sequence. The dashed curve represents the expected course of decline after the first inspection alone. Each solid curve represents hypothetical linear summation of the CAE strength estimates for the first and second inspections. See text for details.

period of inspection, the scores lie near the dashed curve of CAE strength estimates, as expected. Following inspection in SAME mode, addition of the strength estimates predicts increased scores as shown by the upper solid curve. Observed scores for K.W. are transiently increased following the second inspection, in qualitative agreement with the summation rule, but the magnitude of observed increase is always less than that predicted. Scores from tests made the following day (not shown) were not reliably different from the expected strength following the first inspection alone. Results obtained following the SAME mode second inspection do not confirm the linear systems hypothesis.

Consider now the OPPOSITE mode experimental sequence shown by the open points. Prior to the second inspection, the scores again lie near the dashed curve, as expected. Following inspection in OPPOSITE mode, linear summation of the strength estimates predicts decreased scores, as shown by the lower solid curve. Because of the particular convention of displaying the subject's scores (recall that the sign of a score indicates whether green or magenta is predominant on a specific test orientation), addition of complementary CAEs has the net effect of subtracting their strength estimates. At times soon after the OPPOSITE inspection, the subject's scores are expected to change in sign, indicating a qualitative change in the color of $\mathrm{CAE}$ revealed on each test orientation. A few minutes later, the predicted course passes through zero $\mathrm{P}_{\mathrm{e}}$ (indicating achromatic null matches), then gradually returns to predict scores of the original sign but having decreased magnitude (i.e., an apparent "spontaneous" recovery of the original colors of CAEs). Observed scores for subject K.W. show changes over time almost exactly as predicted. The $45^{\circ}$ test grating originally revealed a greenish CAE, which was changed by the OPPOSITE inspection to a pinkish $\mathrm{CAE}$; later, no CAE was noticeable, and still later, the CAE was faintly greenish. Scores from tests made the following day (not shown) indicated the presence of a CAE having the original color, as expected. Results obtained following the OPPOSITE mode second inspection show both qualitative and quantitative changes in color, as predicted on the rule of summation, thus confirming the linear system hypothesis rather strongly.

Figure 3 compares observed scores to the hypothetical curves for subject A.P., showing scores on the index of CAE strength as a function of time after the first inspection. Each plotted point represents the same measures as in Figure 2, the mean $\mathrm{P}_{\mathrm{e}}$ and standard deviation of 10 null match judgments. Closed symbols plot results from the SAME mode sequence in which the interinspection interval lasted $43 \mathrm{~min}$; open symbols show her results from the OPPOSITE mode sequence following an interinspection interval of $40 \mathrm{~min}$. The times at which the second periods of inspection occurred are indicated by arrows, as before. The three smooth curves correspond to those described above. The dashed curve represents typical decay (Equation 5);

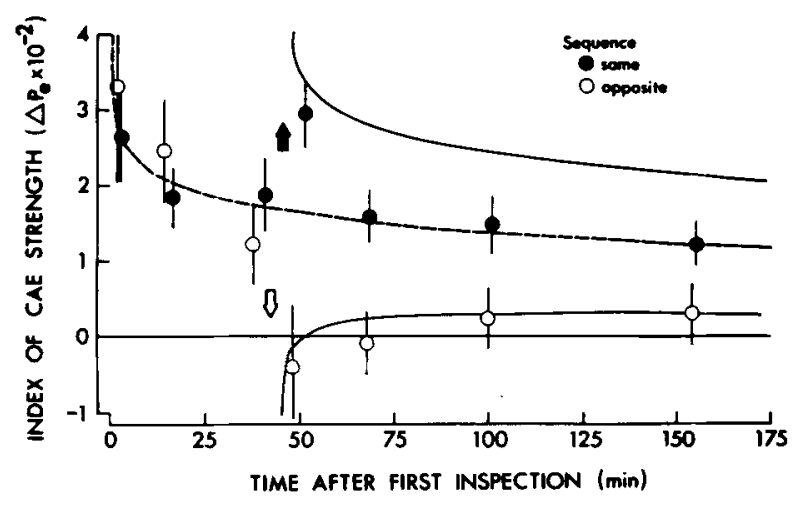

Figure 3. Index of CAE strength shown as a function of time after the first inspection. This graph compares SAME and OPPOSITE mode experimental sequences as described for Figure 2. Results and expectations shown here are for subject A.P. 
upper solid curve, addition (Equation 6a); and lower curve, subtraction (Equation 6 b).

Figure 4 shows findings for subject D.H. Symbols have the same meanings as in the previous figures. His interinspection intervals lasted $94 \mathrm{~min}$ in the SAME mode experimental sequence and $91 \mathrm{~min}$ in the OPPOSITE mode sequence. Curves in Figure 4 correspond to those shown for the other subjects.

Results shown in Figures 2, 3, and 4 are representative of the findings on all subjects for illustrating two main points. First, in the OPPOSITE mode experimental sequences, the subjects' scores were adequately predicted on the linear summation rule. All gave evidence of qualitative changes in the colors of their CAEs as a function of time elapsed after the OPPOSITE mode second inspection, and for all but one subject "spontaneous" recovery of the original CAE colors had persisted through the following day. The exact quantitative predictions were not always as crisply confirmed as shown in the figures, but no evidence of systematic deviations from them was found. Second, in the SAME mode experimental sequences, no subject's scores increased as much as predicted by summation of the CAE strength estimates. The observed increases in scores also showed individual differences; D.H., showed the largest relative increase following the SAME mode second inspection (Figure 4), A.P. showed the smallest relative increase (Figure 3), and $\mathrm{K}$.W. showed an intermediate increase in scores (Figure 2).

\section{Discussion}

The rule of linear summation is confirmed as being a useful one by the results of OPPOSITE mode experimental sequences, but is not confirmed by the results of SAME mode experimental sequences. These discrepant results seem to have two possible explanations.

First, the additivity rule may be adequate for OPPOSITE mode inspections but not adequate for SAME mode inspections because the OPPOSITE inspections are more nearly independent than are the SAME ones. Recall that SAME mode sequences used the identical color-orientation pairings in both periods of inspection. Conceivably, if the first period of inspection produced an alteration in the mechanisms by which those CAEs were established and retained, then the second period of inspection using the identical stimuli could have been rendered less effective. OPPOSITE mode sequences, on the other hand, used the complementary colororientation pairings in the two periods of inspection. Thus, even if the first inspection had produced an alteration in the mechanism by which those original CAEs were established, the complementary stimuli used during the second inspection might establish

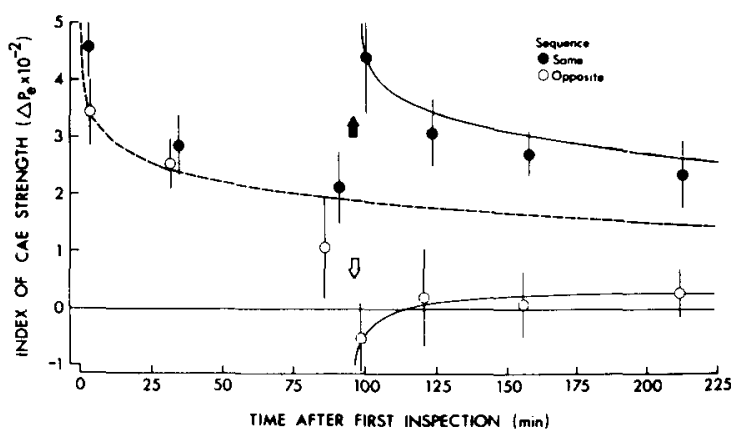

Figure 4. Index of CAE strength shown as a function of time after the first inspection. This graph compares SAME and OPPOSITE mode experimental sequences as described for Figure 2. Results and expectations shown here are for subject D.H.

their own CAEs on a relatively separate, unaffected mechanism. If it were also true that the colorimetric index were numerically linear in relation to strengths, addition of our strength estimates could predict the net behavior for OPPOSITE mode sequences. Yet, the addition rule would fail to predict the net behavior for SAME mode sequences in which the CAE response functions were not independent. In brief, this explanation attributes the lack of linear system behavior to the CAEs themselves rather than to the manner of their assessment.

The second possible explanation for these findings is more readily testable because it attributes the apparent lack of summation to difficulties of assessment. SAME mode inspections increased the subject's net scores, whereas OPPOSITE mode inspections decreased them. The additivity rule might be successful for small, but not for large, scores, because the colorimetric index is nearly linearly related to CAE strengths at low values but not so at high values. In other words, the aftereffects themselves might behave as a linear system for either of the inspection modes, but observations can disclose additive behavior only when the net scores are moderate to low in value. Failure to observe the expected behavior could arise solely from the use of a nonlinear measuring instrument, namely the numerical scores of the subject's color judgments. If this were the sole source of discrepancy, and if scores from both kinds of inspection sequences could be so manipulated as to fall into the nearly linear range on the index (i.e., the specific range over which OPPOSITE mode inspections seem to sum neatly), then SAME mode as well as OPPOSITE mode inspections will yield the expected results. By adopting this as a hypothesis, it is possible to form exact expectations for the outcomes of Experiment 2, which are likewise calculated on the rule of linear summation for the CAE strength estimates. 


\section{EXPERIMENT 2}

In the second experiment, combined CAEs were established by means of multiple periods of inspection carried out as serial reversals of the colororientation pairings. Inspection and testing follow a periodic sequence so that each 5.00 -min period of inspection is followed by a single test session, then by the next $5.00-\mathrm{min}$ inspection and its test, and so on. The initial inspection is of the particular colororientation pairs $45^{\circ}$-magenta, $135^{\circ}$-green. The second inspection is of the OPPOSITE mode, $45^{\circ}$. green and $135^{\circ}$-magenta. The third inspection is of the SAME mode as the initial one $\left(45^{\circ}\right.$-magenta, $135^{\circ}$-green), the fourth inspection is OPPOSITE, the fifth one is SAME. The serial reversals procedure has two aims: (1) to test the summation rule for multiple periods of inspection, and (2) to manipulate the specific range on the colorimetric index upon which net scores fall after SAME mode inspections.

Consider a simplified case in which the factor of CAE decay is neglected. Following the initial $5-\mathrm{min}$ inspection, judgments in the first test session are scored as a value $x$. The second, OPPOSITE inspection is also $5 \mathrm{~min}$ in duration but produces CAEs of the complementary colors (relative to the initial CAEs). The expected effect is to produce a change in scores of the same extent but in the opposite direction on the colorimetric scale. On the rule of summation, continuing for the moment to neglect decay, the $x$ units of increase in score produced by the first inspection are exactly counterbalanced by the $x$ units of decrease produced by the OPPOSITE inspection. Accordingly, judgments in the second test session can be expected to show a net score near zero.

What is the point of asking the subject to inspect twice to get to the zero position on the scale? Recall that the zero position is very nearly the score obtained in the absence of specially produced effects. I ask whether the third 5-min inspection (SAME mode) produces $x$ units of increase in scores, as had been the result of the initial inspection. The reasons that the scores might be the same (in this example) are that the CAEs were established by identical conditions of inspection and they were measured on the identical range of scale (namely between zero and $x$ ). What is different between the initial and the SAME inspections is the recent history of the subject's CAEs, but that fact is irrelevant if the system is a linear one. The summation rule is based on the hypothesis of a linear system, for which the response function is state-invariant.

The example illustrates the rationale of serial reversals of the inspection modes, but is too simplified to represent the exact expectations because it ignores the empirical fact of CAE decay. When the factor of decay is given its appropriate regard, the relevant computations become more tedious. Each partial contribution to a particular test depends on the time that had elapsed between each specific inspection and the test being considered. The algebraic sum of partial contributions on each particular test comprises the expected net score on that test. Because of decay, however, the serial reversals procedure will accomplish only approximately the goal of restricting net scores to the identical range on the colorimetric index. A relevant computational example is given in Table 2.

\section{Method}

Subjects. One male (K.W.) and three females (A.G., J.A., L.G.) participated. Each had previously served as a subject in at least one experiment on CAEs.

Apparatus. The apparatus was the same as that described in Experiment 1.

Procedure. The course of the experimental sequence required 11 steps. (1) The subject was pretested prior to any inspections. (2) Afterward, the initial period of inspection was carried out to build up the first CAEs. (3) The first test session followed the end of inspection in order to assess the strength of the specific CAEs that had just been established. (4) Next, a second period of inspection was carried out in the OPPOSITE mode, to produce a particular net CAE. (5) The second test session which followed assessed the net strength (or combination) of both CAEs that had been established. (6) After the second test session, a third inspection was carried out, in the SAME mode as the initial one. (7) The third test session followed the third inspection in order to assess the combined CAEs built up during all three of the inspections. Following this basic pattern, (8) the fourth inspection was carried out in OPPOSITE mode, (9) the combined CAEs tested a fourth time, (10) then the fifth inspection was in SAME mode, followed by (11) the fifth test session.

The durations of the inspection periods, as well as the specific times for testing, were chosen in a different manner than described for Experiment 1 above. Every period of inspection used here had a duration of $5.00 \mathrm{~min}$. Also, each testing session was made within the first $5.00 \mathrm{~min}$ after the end of the immediately preceding period of inspection. Each of the 11 steps above required $5.00 \mathrm{~min}$ to complete.

\section{Results}

Table 1 compares the results of all four subjects over the series of reversals of the inspection modes. Results are presented as the difference between net scores on sequential test sessions for comparison to the hypothetical expected values. Each entry in the table reports the difference between two values of mean $\mathrm{P}_{\mathrm{e}}$ for specific test judgments, plus or minus one estimated standard error of the difference between means. Since each mean $\mathrm{P}_{\mathrm{e}}$ is based on 10 null match judgments, the difference between two means

Table 1

Differences of Net Scores on Sequential Tests Over the Series of Reversals of the Inspection Modes

\begin{tabular}{|c|c|c|c|c|}
\hline \multirow[b]{2}{*}{ Subjects } & \multicolumn{4}{|c|}{ Differences of Net Scores on Sequential Tests $\left(\mathrm{P}_{\mathrm{e}} \times 10^{-2}\right)$} \\
\hline & $\begin{array}{l}\text { Tests }(1,2) \\
\text { Reversal } 1\end{array}$ & $\begin{array}{l}\text { Tests }(2,3) \\
\text { Reversal } 2\end{array}$ & $\begin{array}{l}\text { Tests }(3,4) \\
\text { Reversal } 3\end{array}$ & $\begin{array}{l}\text { Tests }(4,5) \\
\text { Reversal } 4\end{array}$ \\
\hline $\begin{array}{l}\text { K.W. } \\
\text { A.G. } \\
\text { J.A. } \\
\text { L.G. }\end{array}$ & $\begin{array}{l}4.14 \pm .22(100 \%) \\
2.97 \pm .19(100 \%) \\
3.88 \pm .27(100 \%) \\
6.17 \pm .27(100 \%)\end{array}$ & $\begin{array}{l}2.72 \pm .16(66 \%) \\
2.43 \pm .16(82 \%) \\
2.57 \pm .33(66 \%) \\
4.86 \pm .28(79 \%)\end{array}$ & $\begin{array}{l}2.93 \pm .20(71 \%) \\
2.17 \pm .16(73 \%) \\
2.33 \pm .29(60 \%) \\
4.49 \pm .26(73 \%)\end{array}$ & $\begin{array}{l}2.34 \pm .20(57 \%) \\
2.03 \pm .17(68 \%) \\
2.37 \pm .26(61 \%) \\
3.91 \pm .25(63 \%)\end{array}$ \\
\hline $\begin{array}{l}\text { Mean } \% \text { Observed } \\
\% \text { Expected (Table } 2)\end{array}$ & $\begin{array}{l}100 \\
100\end{array}$ & $\begin{array}{l}73 \\
94\end{array}$ & $\begin{array}{l}69 \\
98\end{array}$ & $\begin{array}{l}62 \\
95\end{array}$ \\
\hline
\end{tabular}


represents 20 judgments. Percentages reported in parentheses compare the relative magnitudes of observed difference scores for each subject. Expected values are given in percentages for ease of comparison (from Table 2).

It is clear that no subject's difference scores are in very close agreement with the hypothetical values. The first reversal of the inspection modes (namely, the initial followed by OPPOSITE inspection) elicits substantially larger difference in scores than the second reversal (OPPOSITE followed by SAME inspections). The SAME mode inspection produced a smaller change in the net CAE strengths than was expected on the rule of linear summation. Subsequent difference scores in the series of inspection mode reversals tend to decline even farther, although in a less dramatic fashion. ${ }^{2}$ These findings do not confirm the rule of linear summation.

\section{Discussion}

The preceding experiments have demonstrated that SAME and OPPOSITE inspections do not obey the same rules. In OPPOSITE inspections, the greenish aftereffects built up initially on $45^{\circ}$ lines can have superposed upon them pinkish aftereffects that were built up subsequently. Following this, the appearance of $45^{\circ}$ test lines was predicted by the simple algebraic sum of appropriate aftereffects measured in isolation. Not so for SAME mode inspections, however. Even though the "causes" (i.e., the inspections) were similarly superposed, the resulting "effects" (i.e., the subjective colorations retained as aftereffects) could not be as readily described. Net scores indicate that CAE strength is in some way less for a

Table 2

A Computational Example of Equation 7 Given in the Appendix

\begin{tabular}{|c|c|c|c|c|c|}
\hline \multirow[b]{2}{*}{ Inspection $\mathrm{i}$} & \multicolumn{5}{|c|}{$\begin{array}{c}\text { Partial Contribution to Scores } \\
\text { of Tests }\left(\mathrm{P}_{\mathrm{e}} \times 10^{-2}\right)\end{array}$} \\
\hline & Test 1 & Test 2 & Test 3 & Test 4 & Test 5 \\
\hline $\begin{array}{l}\text { Initial }(i=1) \\
\text { Opposite }(i=2) \\
\text { Same }(i=3) \\
\text { Opposite }(i=4) \\
\text { Same }(i=5)\end{array}$ & +1.98 & $\begin{array}{l}+1.55 \\
-1.98\end{array}$ & $\begin{array}{r}+1.40 \\
-1.55 \\
+1.98\end{array}$ & $\begin{array}{l}+1.30 \\
-1.40 \\
+1.55 \\
-1.98\end{array}$ & $\begin{array}{l}+1.23 \\
-1.30 \\
+1.40 \\
-1.55 \\
+1.98\end{array}$ \\
\hline \multirow[t]{2}{*}{ Sum (net score) } & +1.98 & -0.43 & +1.83 & -0.53 & +1.76 \\
\hline & & \multicolumn{4}{|c|}{$\begin{array}{l}\text { Sequence of Inspection } \\
\text { Mode Reversals }\end{array}$} \\
\hline \multicolumn{2}{|c|}{ Derived Measure } & $1(1,2)^{*}$ & * $2(2,3)$ & $\begin{array}{l}\text { versal } \\
3(3,4)\end{array}$ & $4(4,5)$ \\
\hline \multicolumn{2}{|c|}{$\begin{array}{l}\text { Difference of Serial Tests } \\
\text { Absolute Value } \\
\% \text { of Largest }\end{array}$} & $\begin{aligned}+2.41 \\
2.41 \\
100\end{aligned}$ & $\begin{array}{l}-2.26 \\
2.26 \\
94\end{array}$ & $\begin{array}{l}+2.36 \\
2.36 \\
98\end{array}$ & $\begin{array}{l}-2.29 \\
2.29 \\
95\end{array}$ \\
\hline
\end{tabular}

Note-Specific parameter values are set in accord with the procedures used in Experiment $2\left(k=1.00 ; D_{i}=5.00 ; t_{i}=a s\right.$ described in text). ${ }^{*}$ Numbers in parentheses indicate number of tests. subsequent inspection in SAME mode than for a corresponding initial one.

The second experiment set out to test the summation rule when combined CAEs were established by a series of reversals of the inspection modes, an advantage being that the subjects' net scores remain within a restricted range on the colorimetric index. In Experiment 1, the target measures were made in different ranges of the colorimetric index, raising the possibility that linear summation was not observed for SAME inspections due to nonlinearity of the measures themselves at high values. In Experiment 2, on the other hand, assessments were made in nearly the same low range of values over which the OPPOSITE inspections of the first experiment had been found to sum neatly. Failure to confirm linear summation in the present results cannot be attributed solely to the numerical scaling of the colorimetric index. Therefore, all these results suggest that OPPOSITE inspections establish more nearly independent CAEs than do SAME inspections, and that a lack of independence for SAME ones plausibly explains their failures to sum.

It may not be surprising that subjects' behaviors after SAME mode inspections are poorly described by linear summation; just on the face of it, perceptual aftereffects would seem so complex as to be impractical to describe with operational equations. Yet this approach has enjoyed success in predicting the outcomes of OPPOSITE inspections. In the next experiment, summation analysis will be applied in further studies of the SAME mode inspections.

\section{EXPERIMENT 3}

The main purpose of the third experiment is to gain a more detailed understanding of the summation failures for SAME mode inspections. Results of the preceding experiments indicate that the initial inspection serves to alter the mechanisms which underlie CAEs so that a subsequent SAME inspection (using the identical stimuli) is rendered less effective. Linear system predictions fail because CAE strengths depend upon the recent history of inspection. The experimental question here asks how the CAEs produced by a SAME inspection depend upon CAEs present initially, to test between alternative forms of dependence. On one hand, SAME mode inspections might be less efficient in building up CAEs because the mechanisms that establish CAE strength came to be altered by the initial aftereffects. On the other hand, the mechanisms that retain CAE strength might do so less efficiently, so that even if the expected amount of strength had been built up, it decays away more rapidly. In their extreme cases, these alternative forms of dependence must lead to distinctive outcomes.

These expectations are less specific than ones for 
the previous experiments. If the main form of dependence is that less strength is established by the SAME inspection, the time course of decay would be that appropriate to the strength actually established. But if the main dependence is an altered rate of decay, the scores will decline over time with extraordinary speed. A body of results obtained in our laboratory (more than 200 decay curves measured) indicates that no matter which parameter of the inspection had been manipulated to vary the initial CAE strengths, the subsequent rates of decline over time follow a remarkably uniform course (Riggs et al., 1974; White, 1976, 1977). Abnormally fast decay can therefore be detected readily.

A specific, and hence more satisfactory expectation can also be tested by this experiment. On the hypothesis that summation failure as observed in Experiment 1 is representative of that presently to be measured, point predictions can be calculated given one key assumption. The calculations require that numerical scores on the colorimetric index are suitably linear as estimates of the CAE strengths. While the results of Experiment 2 demonstrated that nonlinear scaling of the scores cannot account for all of the observed discrepancies, it is here assumed that nonlinearity in the scores is insignificant.

In the third experiment, combined CAEs are established for each subject in two separate experimental sessions. One of the sessions is the control condition, utilizing two successive periods of inspection (initial and OPPOSITE) which differ in certain details from the OPPOSITE mode sequences of Experiment 1 (see below). The other session provides the experimental condition, utilizing three successive periods of inspection. The initial and second inspections of the experimental condition correspond to those used in the control condition, and the third inspection is carried out in SAME mode relative to the initial one.

Experiment 3 seeks measurements comparable to those observed in Experiment 1. Recall that in the SAME mode sequences of the first experiment, the initial inspection was followed 90 min later by the SAME inspection. The experimental condition used here will repeat that specific arrangement, but with the proviso that a period of OPPOSITE inspection intervenes between the initial and the SAME ones. The OPPOSITE inspection is used to reduce the subjects' net scores in a manner similar to that for Experiment 2, but here it is used to establish a very specific net CAE. The particular parameters of the OPPOSITE inspection are chosen in such a way that the subjects' net scores for certain tests (those made $90 \mathrm{~min}$ or longer after the initial inspection) have expected values very near zero in the control condition. The experimental condition starts out like the control condition, but about 90 min after the first inspection a third inspection in SAME mode is carried out (as in Experiment 1). Tests made after the SAME inspection provide the evidence of particular concern; the extent and persistence of CAE strength increase.

\section{Method}

Subjects. One male (K.W.) and one female (H.B.) served as subjects. These individuals were selected from the subjects of Experiment 1 because their scores were highly similar, large in value and reliable. The similarity of scores facilitates direct comparison of their performances.

Apparatus. Same as that used in Experiment 1.

Procedure. Each subject participated individually in two conditions (control and experimental), which were carried out in different weeks. The control condition consisted of five steps: (1) A pretest session; (2) the first period of inspection (11.25 min); (3) an interinspection interval lasting $38.75 \mathrm{~min}$, during which three test sessions were made; (4) the second inspection in OPPOSITE mode (6.25 min); and (5) a sequence of test sessions made after the second inspection. The main purpose of the control condition was to establish the initial CAEs with the 11.25 -min inspection, assess them, then establish oppositely colored CAEs with $6.25 \mathrm{~min}$ OPPOSITE inspection. The sequence of assessments made afterward were to show whether net test scores were near zero at the appropriate times after inspection, as desired.

The experimental condition incorporated the first four steps of the control condition into a seven-step sequence. In new Step 5, only three or four test sessions were made after the OPPOSITE inspection. In Step 6, a third inspection in SAME mode lasting $5.00 \mathrm{~min}$ was carried out. In Step 7, a sequence of test sessions was made after the third period of inspection had ended. The amounts of time that elapsed between the end of the first inspections and the beginnings of the third inspections were $90 \mathrm{~min}$ for subject K.W., and $85 \mathrm{~min}$ for subject H.B. These values were comparable to the interinspection intervals of Experiment 1 for these particular subjects.

\section{Results}

Figure 5 compares results of the experimental condition to those of the control condition for both subjects. Test scores are shown on the index of CAE strength (in units of $\mathrm{P}_{\mathrm{e}} \times 10^{-2}$ ) as a function of the time elapsed after the first inspection. Each plotted point represents the mean $P_{e}$ for 10 null match judgments, made at the mean time shown on the abscissa. The mean $\mathrm{P}_{\mathrm{e}}$ of each subject's pretest judgments was subtracted out before plotting. Vertical bars mark off one standard deviation of the test scores on either side of their mean. Different symbol shapes represent the different subjects (circles, K.W.; triangles, H.B.). Open' symbols plot results from the control conditions, while closed symbols plot those from the experimental conditions.

Consider the open symbols which show scores in the control condition. The initial period of inspection produced a change in scores that is qualitatively and quantitatively like that found in Experiment 1. The second period of inspection (in OPPOSITE mode) was carried out at the time indicated by the open arrow. Tests made following the second in- 


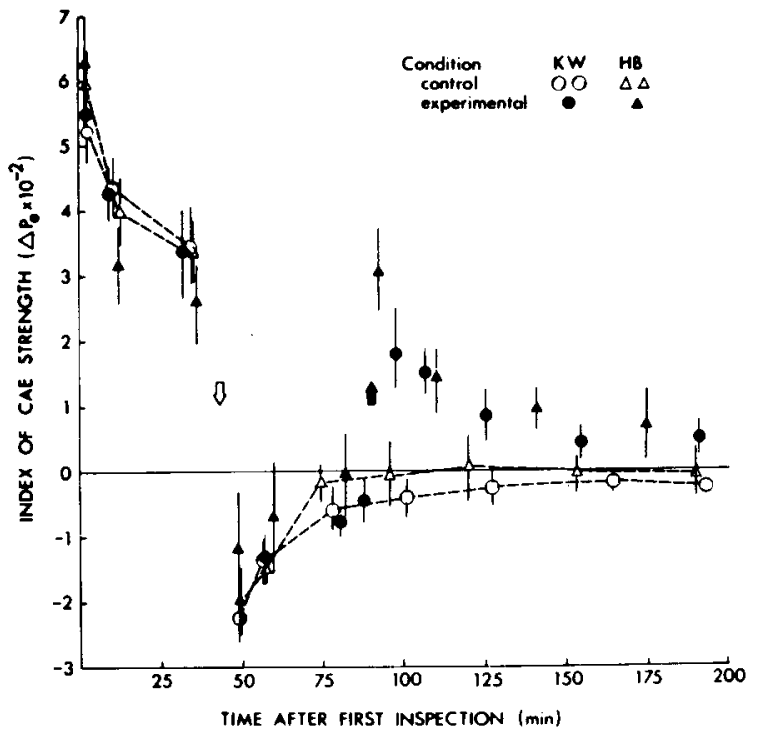

Figure 5. Index of CAE strength shown as a function of time after the first inspection. This graph compares control and experimental conditions as described for Experiment 3 in text. The time of occurrence for the second (OPPOSITE) inspections used in both control and experimental conditions are indicated by the open arrow on the figure. The time of occurrence for the third (SAME) inspections used in the experimental condition are indicated by the filled arrow. Open symbols plot the results from the control conditions, while filled symbols plot those from the experimental conditions. Different symbol shapes represent the different subjects $(O, K . W . ; \Delta$, H.B. $)$

spection show net scores that are initially negative, indicating a reversal of the apparent coloration of the CAEs, and at later times tend to lie near zero $P_{e}$ as expected (namely, for tests made $90 \mathrm{~min}$ or longer after the end of the first inspection).

Consider now the closed symbols which show scores in the experimental condition. Inasmuch as the first several steps of procedure correspond to the control condition, results from tests made during the first $90 \mathrm{~min}$ after the first inspection are expected to replicate the control condition. The open and closed circles, results for K.W., do trace out nearly identical net scores over that interval. The open and closed triangles, results for H.B., do not overlap to the same extent as do those for K.W. but nevertheless are similar. In the experimental condition, a third period of inspection (in SAME mode) was carried out at the time indicated by the filled arrows. Closed symbols to the right of the arrow plot the net scores following all three inspections. It is clear that the SAME inspection produced a transient increase in the subjects' net scores, with both of the subjects showing highly similar trends in their results.

Figure 6 compares the experimental findings to the expectations. The dependent measure in this graph is a difference score, shown in units of $\mathrm{P}_{\mathrm{e}} \times 10^{-2}$, derived as detailed below. The difference score is a measure of change produced by the experimental treatment. Difference scores are shown as a function of the time elapsed after the SAME mode inspections, so as to display the extent and persistence of the increases in scores they produced.

Different symbol shapes in Figure 6 represent measures from different subjects (circles, K.W.; triangles, H.B.). Closed symbols plot results of the present experiment (initial, OPPOSITE, then SAME inspections). These particular difference scores can be derived by measuring off the distance between the appropriate dotted line and the filled points that are shown in Figure 5, or equivalently by subtracting the control condition score from the experimental condition score at the same specific times after inspection. Open symbols plot difference scores obtained from the results of Experiment 1 (initial then SAME inspections). Open circles (for K.W.) can be derived by measuring off the distance between the dotted smooth curve and the filled points shown in Figure 2. Each measure represents the difference between the expected score had there been no SAME inspection and that observed when the SAME inspection was carried out. The open triangles (for H.B.) were derived in the same manner from results not presented here.

The smooth curve in Figure 6 traces the expected results for a 5.00 -min inspection carried out alone (or as the first period of inspection). Data compared to it, of course, were found after 5.00-min SAME mode inspections so that the subjects had a recent history of CAEs. The comparison indicates that less CAE strength was found even on the earliest tests made, and also indicates a rapid decline in assessed strength with time. The difference scores (and also

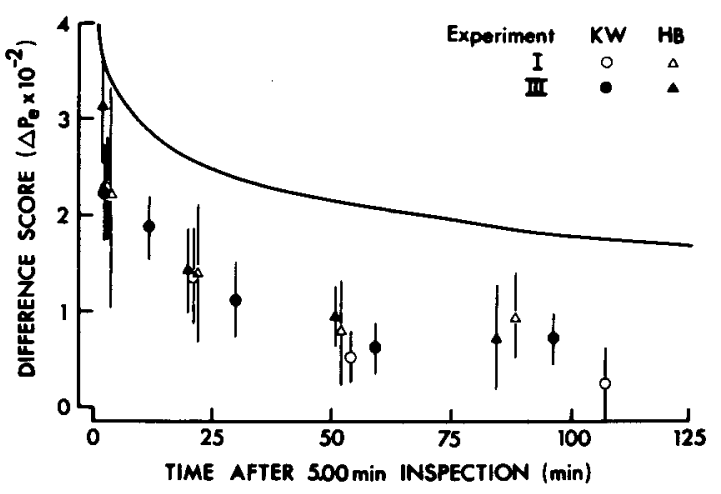

Figure 6. Difference score shown as a function of time after the 5.00-min SAME inspection. The difference scores are derived measures used to indicate the change produced by the SAME inspection. The derived measures facilitate comparing the effects following SAME inspection in Experiment 1 (open points) with the corresponding effects in the experimental condition of Experiment 3 (filled points). Different symbol shapes represent the different subjects $(O, K . W . ; \Delta$, H.B. $)$. The smooth curve represents hypothetical linear summation of the CAE strength estimates. 
the raw scores in Figure 5) have declined nearly to zero within 90 min after the SAME mode inspection, whereas for a typical CAE of the same initial strength, such decline would require at least a full day.

The closed symbols and the open ones in Figure 6 are in close agreement, apparently confirming the specific expectation that CAEs established by SAME mode inspection sum in a similar nonlinear manner in both Experiments 1 and 3. The changes in scores produced by SAME inspection were nearly identical, without regard either to procedural differences (i.e., the intervening OPPOSITE inspection of Experiment 3 ) or to the absolute values of the scores. Recall that in Experiment 1 the target measures were obtained at high numerical scores, but in the present experiment at low numerical scores. The ability to translate the target measures from one scale range to another demonstrates that the numerical scale itself is suitably linear.

\section{DISCUSSION}

The findings of Experiment 3 show that a SAME inspection establishes CAEs that are qualitatively distinct from those built up by an isolated or initial period of inspection. Most importantly, the decline with time of assessed CAE strength is extraordinarily rapid, arguing rather strongly that the mechanisms which retain CAE strength come to be altered by a history of inspection. Neither shortening the duration of inspection nor degrading the luminance, contrast, or excitation purity of the inspection patterns yields results like these (White, 1977). Present findings therefore demonstrate the lack of a crucial feature of linear systems, namely a stateinvariant response function.

\section{CONCLUSIONS}

The aims of these three experiments have been both practical and theoretical. One practical question concerns use of OPPOSITE inspection as a "neutralization" procedure to eliminate CAEs (e.g., Skowbo et al., 1974, 1975; Stromeyer, 1969). Results of all three experiments raise serious doubts, offering quantitative evidence on the failures of "neutralization" as observed in other laboratories (Siegel \& Nachmias, 1975; Smith, Note 4). The "neutralization" technique relies on the assumption that greenish and pinkish CAEs for a particular orientation cancel in a way that leaves no residual aftereffect. Results here are inconsistent with that assumption, suggesting rather than CAEs of complementary colors are retained independently.

Also of practical interest is the successive inspections paradigm and the corresponding analysis of summation properties. Clearly, SAME and OPPOSITE modes of inspection are merely examples of the possible ways to test for interactions between aftereffects. Preliminary work has made use of achromatic patterns in one of the inspection periods so as to study the "interference" caused by achromatic inspection (Skowbo et al., 1974; White \& Graves, Note 5). Other preliminary work has made use of gratings that differ in orientation between inspections, the extent of interaction providing a measure of orientation-tuning (Ellis \& Castellan, Note 6). Additional possibilities are easily conceived.

The theoretical impact of these experiments arises from quantitative questions. The ability to devise models of CAEs is constrained by not knowing in what manner the strengths of the CAEs per se are represented by the scores assigned empirically. The state of the nervous system that is the actual aftereffect cannot now be measured directly in order to validate the numerical scale of scores. But it is possible to argue for the plausibility and parsimony of the scale used here to represent CAE strength.

Three experiments have asked whether useful estimates of CAE strengths can be made from scores on this particular colorimetric index. They have tested for predictive validity on the hypothesis that CAEs and the assessments of them behave as a linear system. A fundamental property of linear systems is the superposition of causes and effects, and it is this hypothetical property that allows specific predictions to be calculated for successively established CAEs. In practice, the experiments have combined successive periods of inspection, each inspection being the "cause" of particular CAEs, and attempted to measure the net CAE strengths which were the combined "effects." Evidence showed that the mechanisms which retain each specific CAE are influenced by prior inspection and hence do not behave as a linear system. For OPPOSITE inspections, agreement with the linear system predictions can be attributed to the independence of mechanisms responsible for the combined CAEs. Yet it must also be attributed to certain useful properties of the colorimetric index used for assessment. Would the point predictions have had success if the numerical scores on this index were not suitably linear estimates of the CAE strengths?

The central goal of this research is to describe CAEs through an operational approach that is akin to linear systems analysis. Comprehensive studies of this kind are expected to clarify the relative contributions of perceptual and learning processes to these aftereffects, and to provide important groundwork toward identifying their physiological bases. Even the elementary data given here may point to a fruitful direction for future models. Is it really useful to categorize CAEs as being examples of visual 
physiology vs. being examples of learning or memory-like processes? Could not such attributes as "spontaneous recovery," predicted here by summation equations, be likewise derived from theories of learning? Clearly, at some point in the nervous system the distinction between the physiology of vision and that underlying learning or memory must break down. Conceivably, the physiological mechanisms of pattern vision (on the other hand) and the factors of learning and memory (on the other) are just the extremities of a common corpus from which CAEs derive their distinguishing features.

\section{APPENDIX I}

Quantitative expectations based on the time courses for establishment and decay of CAEs, used to test for linear system-like behavior. Riggs et al. (1974) used a colorcancellation procedure similar to that used here and found that measurements of the colorimetric purity ${ }^{3}$ that subjects judged to be a null match decreased nearly linearly as a function of the logarithm of time after inspection had ended. Examples of such data are shown in Riggs et al. (1974) (p. 538) and in Figure 1 of the present paper. Such decay is well described by an equation of the form:

$$
\Delta \mathrm{P}_{\mathrm{e}}=\mathrm{a}-\mathrm{b} \ln \mathrm{t} \quad \text { for } \mathrm{t}>1,
$$

where $\Delta \mathrm{P}_{\mathrm{e}}=$ change in excitation purity ${ }^{5}$ of subjects' null matches, $a=$ value of $P_{e}$ at $t=1$ min (i.e., $1 \mathrm{nt}=0$ ), $b=$ slope constant ("rate of decay"), and $t=$ time after inspection ( $\mathrm{min}$ ).

Equations of this form were fitted to more than 200 decay curves, by the method of least squares. The regression constants, a ("initial strength") and b ("rate of decay"), are highly correlated, without regard to the identity of individual subjects or to the specific conditions of inspection. Specifically:

$$
\mathrm{b}=.12 \mathrm{a} .
$$

By substitution of Equation 2 into Equation 1:

$$
\Delta \mathrm{P}_{\mathrm{e}}=\mathrm{a}(1-.12 \ln \mathrm{t}) .
$$

Riggs et al. (1974) noted a monotonic increasing dependence of the "initial strength" (regression constant a) on the duration of the inspection period which had been used to build up the aftereffects. Over an intermediate range of inspection durations (such as those used in the present study), this dependence was approximated by:

$$
\mathrm{a}=\mathrm{kD}^{0.5} \text {, }
$$

where $\mathrm{k}=\mathrm{a}$ constant of proportionality whose value is characteristic for each subject, and $D=$ duration of the inspection period $(\mathrm{min})$. By substitution of Equation 4 into Equation 3,

$$
\Delta \mathrm{P}_{\mathrm{e}}=\mathrm{kD}^{0.5}(1-.12 \ln \mathrm{t}) .
$$

Equation 5 provides a simple description which is partially adequate in summarizing the effects from two independent variables (D, duration of the inspection period, and $t$, time after inspection when tests were made). Note that in Equation 5 there is one free parameter for calculating the expected values of $\Delta \mathrm{P}_{\mathrm{e}}$ during the time course of decay for any given individual. The free parameter is $k$, a constant of proportionality whose value is characteristic of each subject and accordingly must be estimated.

The present experiments used two periods of inspection which occurred with relatively short delays between them. The particular test sessions which take place during the interinspection interval assess the CAEs built up by the first inspection. Results of those tests are used to estimate each subject's specific factor of proportionality $(k)$ in the following manner: (a) The mean $P_{e}$ and mean time after inspection were calculated for each test session made during the interinspection interval; (b) for each mean time after inspection, a relative expected value was calculated from Equation 5 by setting the values $\mathrm{k}=1.000, \mathrm{D}=11.25$, $\mathrm{t}=$ the value observed for mean time of test session; (c) each observed value of mean $P_{e}$ from (a) was divided by the appropriate relative expected value calculated in (b); and (d) the geometric mean of the ratios calculated in (c) was taken as the estimated value of $\mathrm{k}$ for that particular subject.

The empirical estimates so derived were: for subject H.B., $k=1.83$; for K.W., $k=1.78$; for D.H., $k=1.24$; for A.P., $k=0.92$.

The rule to be tested in Experiment 1 was that the net aftereffects established by two successive inspections could be predicted by the algebraic sum of two appropriate examples of Equation 5. If the second period of inspection was of the SAME color-orientation pairs as the first inspection, the expected curve following the second inspection had the form shown in Equation 6a. If the second period of inspection was of OPPOSITE color-orientation pairs, the expected curve following the second inspection had the form shown in Equation $6 b$.

$$
\Delta P_{e}=k\left[D_{1}{ }^{0.5}\left(1-.12 \ln t_{1}\right)+D_{2}^{0.5}\left(1-.12 \operatorname{lnt} t_{2}\right)\right]
$$

$$
\Delta \mathrm{P}_{\mathrm{e}}=\mathrm{k}\left[\mathrm{D}_{1}^{0.5}\left(1-.12 \ln \mathrm{t}_{1}\right)-\mathrm{D}_{2}{ }^{0.5}\left(1-.12 \ln \mathrm{t}_{2}\right)\right],
$$

where $\mathrm{k}=\mathrm{a}$ constant of proportionality whose value is estimated for each subject, $D_{1}=$ duration of first inspection $(\mathrm{min}), \mathrm{t}_{1}=$ time after first inspection $(\mathrm{min})$, $\mathrm{D}_{2}=$ duration of second inspection $(\mathrm{min})$, and $\mathrm{t}_{2}=$ time after second inspection ( $\mathrm{min}$ ).

In order to calculate expected values when multiple periods of inspection are used, the basic equations shown in (6a) and (6b) are linked to form an appropriate sequence. The general form used in such calculations is shown in Equation 7. In this equation, the expected score on a particular test $(T)$ is shown as the sum of partial components. Each partial component arose from a preceding period of inspection, its value on test $T$ being a joint function of the duration of each inspection period 
$\left(D_{i}\right)$ and the time that had elapsed between the end of each inspection and the test $T\left(t_{i}\right)$. The sign of each partial contribution depends on the mode of its particular inspection, in the same manner as described above.

$$
P_{e}(T)=k\left[\sum_{i=1}^{n} \pm D_{i}^{0.5}\left(1-.12 \ln t_{i}\right)\right] \quad \text { for } t_{i} \geqslant 1 \text {, }
$$

where $i=1,2,3, \ldots, n, D_{i}=$ duration of the $i^{\text {th }}$ inspection $(\min ), t_{i}=$ time elapsed between the end of the $i^{\text {th }}$ inspection and the particular test $T$, and + or - depends on the mode of the $i^{\text {th }}$ inspection relative to the first inspection.

Table 2 shows a computational example which details how the expected values for Experiment 2 result from linear summation. The upper part of the table gives calculated values for the partial contributions which are attributed to each preceding inspection (i). The lower part of Table 2 shows particular derived measures that are based on the difference of scores on two successive tests, expected to be nearly constant on the hypothesis of linear summation. Difference scores are converted to percentage values as these do not depend on the value of $k$ characteristic of the subject, hence percentage expectations are readily applied for different individuals.

\section{REFERENCE NOTES}

1 Montalso F. 5 th associative learning model of the

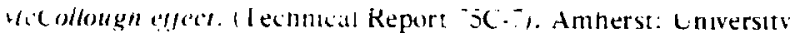
of Massachusetts. Department of Computer and Information Science, 1975.

2. Harris C. S. Insight or out of sight?: Two examples of perceptual plasticity in the human adult. Chapter to appear in C. S. Harris (Ed.), Visual coding and adaptability. Book in preparation, 1977

3. Stromeyer, C. F. Form-color aftereffects in human vision. Chapter to appear in H. Teuber \& R. Held (Eds.), Handhook of sensory physiology (Vol. 8). Book in preparation, 1977.

4. Smith. M. The McCollough effect: Application of leaming theon' to physiology. Unpublished manuscript. Department of Psychology, Dalhousie University, 1974.

5. White, K. D., \& Graves. A. L. Intluence of achromatic inspection on the McCollough effect. Paper presented at the meeting of the Association for Research in Vision and Ophthalmology. Sarasota. Florida, May 1976.

6. Ellis, S. R., \& Castellan, K. Orientation specificity of the McCollough effect: An equivalent-contrast transformation. Paper presented at the meeting of the Association for Research in Vision and Ophthalmology, Sarasota. Florida, April 1975.

\section{REFERENCES}

Anstis. S. M. What does visual perception tell us about visual coding? In M. S. Gazzaniga \& C. Blakemore (Eds.). Handbook of psychobiology. New York: Academic Press, 1975.

Brindey. G. S. Physiology of the retina and visual pathway. Baltimore: Williams \& Wilkins, 1970.

COMmitTeE ON COLORIMETRY, Optical Society of America. The science of color. New York: Crowell. 1953.

Creutzfeldt, $O$. D. \& Heggelund, P. Neural plasticity in visual cortex of adult cats after exposure to visual patterns. Science. 1975. 188. 1025-1027.

Evans. R. M. An introduction to color. New York: Wiley. 1948.
Fidell, L. S. Orientation specificity in chromatic adaptation of human "edge-detectors." Perception \& Psychophysics, 1970, 8. 235-237.

GRAHAM, C. H. (Ed.) Vision and visual perception. New York: Wiley, 1965.

Harris, C. S., \& Gibson, A. R. Is orientation-specific color adaptation in human vision due to edge-detectors, afterimages, or "dipoles"? Science, 1968, 162, 1506-1507.

MacKay, D. M., \& MacKay, V. The time course of the McCollough effect and its physiological implications. Journal of Physiology, 1974, 237, 38-39P.

MACKAY, D. M.. \& MACKAY, V. Dichoptic induction of McCollough-type effects. Quarterly Journal of Experimental Psychology, 1975, 27, 225-233.

MaYheW, J. E. W., \& ANstis, S. M. Movement aftereffects contingent on color, intensity, and pattern. Perception \& Psychophysics, 1972, 12, 77-85.

McCollough, C. Color adaptation of edge-detectors in the human visual system. Science, 1965, 149, 1115-1116.

Murch, G. M. Classical conditioning of the McCollough effect: Temporal parameters. Vision Research, 1976, 16, 615-619.

Riggs, L. A., White, K. D., \& Eimas, P. D. Establishment and decay of orientation-contingent aftereffects of color. Perception \& Psychophysics, 1974, 16, 535-542.

Siger, C., \& Nachmias, J. A re-evaluation of curvature-specific chromatic aftereffects. Vision Research, 1975, 15, 829-836.

Skowbo, D., Gentry, T., Timney, B., \& Morant, R. B. The McCollough effect: Influence of several kinds of visual stimulation on decay rate. Perception \& Psychophysics, 1974. $16,47-49$.

Skoweo, D., Tumey, B. N., Gentry, T. A., \& Morant, R. B McCollough effects: Experimental findings and theoretical accounts. Psychological Bulletin, 1975, 82, 497-510.

Stromeyer. C. F. Further studies of the McCollough effect. Prrimin \& Psychophusics. 1969, 6. 105-110.

WhITE, K. D. Luminance as a parameter in establishment and testing of the McCollough effect. Vision Research, 1976. 16. 297.302.

White, K. D. Studies of form-contingent color aftereffects. In J. Armington, J. Krauskopf, \& B. Wooten (Eds.) Visual psychophysics: Its physiological basis. New York: Academic Press, 1977, in press.

Wyszecxi, G., \& Strles, W. S. Color science: Concepts and methods, quantitative data and formulas. New York: Wiley. 1967.

\section{NOTES}

1. In supplementary control experiments, the Dove prism was removed and inspection pattern orientations changed by hand, with no substantial difference in the results.

2. In supplementary experiments, I have carried out longer series of reversals of the inspection modes. Preliminary results on one subject (K.W.) indicate that difference scores comparable to those reported in Table 1 continue to decline over at least 27 reversals of the inspection modes. In that experiment, K.W.'s difference scores (ait units of $P_{e} \times 10^{-2}$ ) were: 1st reversal, 4.18I $0.23 ; 27$ th reversal, $1.84 \pm 0.21(44 \%)$.

3. Unlike the case with excitation purity, the maximum attainable values for colorimetric purity depend on the values of dominant wavelength and on the particular illuminant. Colorimetric purity is, in practice, a more readily measured specification, being defined by the relation:

$$
P_{c}=\frac{L_{\lambda}}{L_{k}+L_{\omega}}
$$

where $P_{c}$ is the colorimetric purity, $L_{\lambda}$ is the luminance of the 
monochromatic component, and $\mathrm{L}_{\omega}$ is the luminance of the achromatic component provided by the illuminant. Colorimetric purity can be transformed into excitation purity by the use of the formula (see Committee on Colorimetry, O.S.A., 1953, $297 \mathrm{ff}$ ):

$$
P_{e}=\frac{P_{c}}{e-f\left(P_{c}\right)},
$$

where $P_{e}$ is the excitation purity, $P_{c}$ is the colorimetric purity, and $e, f$ are constants whose values depend on dominant wavelength and on the specific illuminant.

(Received for publication March 9, 1977; revision accepted May 23, 1977.) 